\title{
Can the Wish to Receive Intensive Treatment in Elderly Patients with Respiratory Tract Infection Be Predicted?
}

\author{
Shuichi Hagiwara ${ }^{1,2}$, Minoru Kaneko ${ }^{1}$, Makoto Aoki ${ }^{1,2}$, Masato Murata ${ }^{1,2}$, Yumi Ichikawa ${ }^{1,2}$, \\ Jun Nakajima ${ }^{1,2}$, Yuta Isshiki ${ }^{1,2}$, Yusuke Sawada ${ }^{1,2}$, Jun'ichi Tamura ${ }^{2,3}$ and Kiyohiro Oshima ${ }^{1,2}$
}

\begin{abstract}
:
Objective Almost no Japanese elderly patients have an advance directive (AD). Our aim was to determine whether or not the wish to receive intensive care in elderly patients with respiratory tract infection could be predicted from the prehospital data.

Methods In this retrospective study, we reviewed patients $\geq 65$ years of age with respiratory tract infection who had been transferred to our hospital by ambulance between September 2014 and August 2016. The patients were divided into two groups according to whether or not they wished to receive intensive treatment. We placed patients without a wish to receive intensive treatments (WITs) in Group A and patients with a WITs in Group B. We then analyzed parameters that could be determined in the prehospital phase and compared the findings between the groups.

Results Thirty-seven patients were in Group A, and 67 patients were in Group B. None of the patients in this study had an AD. There were significant differences in the age, rate of residence in an extended care facility, frequency of inability to care for oneself fully, frequency of dementia, number of prescribed drugs, and Glasgow coma scale (GCS) on a univariate analysis. A logistic regression analysis showed that the inability to care for oneself fully [odds ratio (OR): $4.521,95 \%$ confidence interval (CI): 2.024-10.096, $\mathrm{p}<0.001$ ] and a low GCS (OR 0.885, 95\%CI 0.838-0.935, p<0.001) were related to a WITs.

Conclusion Elderly patients who are unable to care for themselves and who have a low GCS in the prehospital stage are likely not to want intensive treatment.
\end{abstract}

Key words: elderly, respiratory tract infection, living will

(Intern Med 57: 1989-1993, 2018)

(DOI: 10.2169/internalmedicine.0155-17)

\section{Introduction}

Japan is a super-aged society. In Japan, the emergency transport of elderly people is increasing (1). Two features of the emergency situation for elderly Japanese patients are as follows: First, few elderly patients have an advance directive (AD) or an advance care plan. Fukuhara (2) reported that the author had treated only 1 patient who had an AD out of over 500 patients with an intracranial disorder at a hospital in Japan. Second, many elderly patients die because of respiratory tract infection (RTI); indeed, in Japan, RTI is the third leading cause of death (3). Aspiration pneumonia is also common in elderly people (4). Artificial nutrition and hydration are common in the treatment and care of Japanese elderly patients with deglutition disorder. This is not completely unrelated to causes of death among elderly people.

Many elderly patients with an RTI are transferred via emergency medical transport from a care facility $(\mathrm{CF})$ because of the absence of an AD. However, many elderly patients actually do not want to receive intensive treatment (3), prompting medical staff members to worry that these patients may occupy intensive-care units of hospitals despite not wanting such treatment, displacing other patients who both want and need that treatment. Japanese emergency medical services often transport elderly patients with an RTI

${ }^{1}$ Department of Emergency Medicine, Gunma University Graduate School of Medicine, Japan, ${ }^{2}$ Emergency Medical Care Center, Gunma University Hospital, Japan and ${ }^{3}$ Department of General Medicine, Gunma University Graduate School of Medicine, Japan Received: September 3, 2017; Accepted: November 26, 2017; Advance Publication by J-STAGE: March 9, 2018 Correspondence to Dr. Shuichi Hagiwara, shuhagiwara@gunma-u.ac.jp 


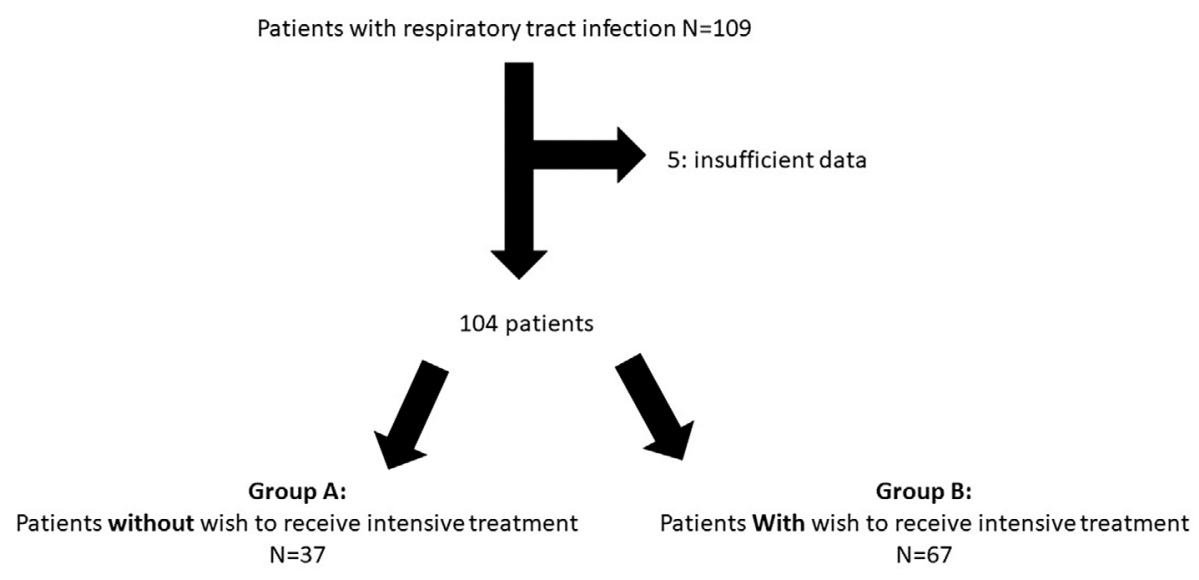

Figure. The study flow diagram.

to a high-quality medical center because of the absence of an $\mathrm{AD}$, even though many Japanese people do not want excessive medical treatment at the end of their life (3).

Given the above, we studied whether or not the wish to receive intensive treatment (WIT) could be predicted from the prehospital data in order to reduce the unnecessary transport of elderly people with an RTI.

\section{Materials and Methods}

This single-center, retrospective, observational study was conducted at Gunma University Hospital. The protocol of this study was approved by the research ethics board of The Gunma University Hospital (Maebashi, Gunma, Japan) (No. 1442).

We reviewed the medical records of patients $\geq 65$ years of age with an RTI who were transferred to Gunma University Hospital by ambulance between September 2014 and August 2016. Patients were divided into two groups according to their WIT. We placed patients without a WIT in Group A and those with a WIT in Group B. We defined "intensive treatment" as transfer to the intensive-care unit, resuscitation, and equipment with a new medical appliance, such as a respirator, pacemaker, cardiac defibrillator, and blood purification therapy. In this study, each patient's therapy was decided by the attending doctors and the patient's own wish or the patient's wish as described by close family members. We analyzed the data that could be determined in the prehospital phase: the age, gender, whether the patient lived at home or in a $\mathrm{CF}$, presence of dementia, whether or not the patient could fully care for themselves, number of prescribed drugs (e.g., drugs for hypertension or diabetes), hospitalization within one year, previous illnesses that affected the activity of daily life (ADL), and prehospital vital signs [mean blood pressure $(\mathrm{mBP})$, heart rate $(\mathrm{HR})$, respiratory rate $(\mathrm{RR})$, body temperature (BT), Glasgow coma scale (GCS), and $\mathrm{SpO}_{2}$ ]. We also compared between the two groups the parameters that were able to be determined after the arrival: the sequential organ failure assessment score (SOFA) (5), quick SOFA (qSOFA) (6), and the score of the Japanese Association for
Acute Medicine (JAAM) for disseminated intravascular coagulation (DIC) (7). Furthermore, we analyzed the inhospital mortality and existence of an $\mathrm{AD}$, the presence of aspiration pneumonia, and the rate of the patient being the decision-maker regarding their own well-being.

\section{Statistical analyses}

Age is shown as the mean ( \pm standard deviation). Differences between the two groups were analyzed with the Mann-Whitney U test for continuous variables and Fisher's exact test for categorical variables. We conducted a logistic regression analysis to detect independent variables for the WIT. When we conducted this logistic regression analysis, we selected independent variables that could be determined during the prehospital phase and had significant differences in the univariate analysis as candidates. The IBM SPSS Statistics 24 software program (IBM Corporation, Endicott, USA) was used for the statistical analyses. $p$ values of less than 0.05 were considered significant.

\section{Results}

Figure shows the study flow diagram. One hundred and nine patients were transported by ambulance to our hospital and admitted during the study period because of an RTI. Five patients were excluded because of insufficient data. We ultimately analyzed 104 patients. Among these 104 patients, 37 did not wish to receive intensive treatment (Group A), and 67 wished to receive intensive treatment (Group B). No patients in this study had an AD.

The results of the univariate analyses are shown in Ta-

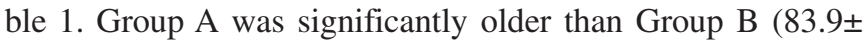
8.1 years vs. $80.2 \pm 7.3$ years, $\mathrm{p}=0.011$ ). A significantly greater percentage of Group A patients lived in a CF $[45.8 \%$ $(17 / 37)$ in Group A vs. 25.4\% (17/67) in Group B, p< $0.001]$. The percentage of patients unable to fully care for themselves was $86.5 \%$ (32/37) in Group A and 40.3\% (27/ 67 ) in Group B, showing a significant difference $(\mathrm{p}<0.001)$. A significantly higher percentage of patients in Group A had dementia [78.4\% (29/37) in Group A, and 46.3\% (31/67) in 
Table 1. Patients' Characteristics.

\begin{tabular}{lccr}
\hline & Group A (n=37) & Group B $(\mathrm{n}=67)$ & p value \\
\hline Age (years old) & $83.9 \pm 8.1$ & $80.2 \pm 7.3$ & 0.011 \\
Male [n (\%)] & $20(54.1)$ & $39(58.2)$ & 0.686 \\
Resident of a CF [n (\%)] & $17(45.8)$ & $17(25.4)$ & $<0.001$ \\
Need care [n (\%)] & $32(86.5)$ & $27(40.3)$ & $<0.001$ \\
With dementia [n (\%)] & $29(78.4)$ & $31(46.3)$ & 0.002 \\
Hospitalization within 1 year [n (\%)] & $13(35.1)$ & $11(16.4)$ & 0.05 \\
Previous illness [n (\%)] & $19(51.4)$ & $19(28.4)$ & 0.033 \\
Addictive medicine (n) & $7.3 \pm 3.9$ & $5.6 \pm 3.8$ & 0.027 \\
mBP (mmHg) & $88.6 \pm 22.9$ & $92.8 \pm 21.4$ & 0.330 \\
Hart rate (min $\left.{ }^{-1}\right)$ & $100.3 \pm 24.7$ & $98.0 \pm 22.2$ & 0.964 \\
Respiratory rate (min $\left.{ }^{-1}\right)$ & $27.2 \pm 6.5$ & $26.2 \pm 9.2$ & 0.169 \\
Body temperature $\left({ }^{\circ} \mathrm{C}\right)$ & $37.9 \pm 1.2$ & $37.8 \pm 1.4$ & 0.958 \\
GCS & $11.3 \pm 3.9$ & $12.6 \pm 3.4$ & 0.040 \\
SpO $(\%)$ & $86.1 \pm 10.5$ & $87.8 \pm 8.2$ & 0.490 \\
qSOFA & $1.5 \pm 0.8$ & $1.3 \pm 0.8$ & 0.484 \\
SOFA & $4.4 \pm 1.8$ & $4.0 \pm 2.7$ & 0.054 \\
JAAM DIC score & $1.5 \pm 1.6$ & $1.2 \pm 1.3$ & 0.478 \\
Aspiration pneumonia [n $(\%)]$ & $18(48.6)$ & $18(26.9)$ & 0.032 \\
Decision maker: patient [n $(\%)]$ & $2(5.4)$ & $24(35.8)$ & $<0.001$ \\
Death [n $(\%)]$ & $6(16.2)$ & $5(7.5)$ & 0.193 \\
\hline
\end{tabular}

Group A, did not have a wish to receive intensive treatment; Group B, wished to receive intensive treatment.

SD: standard deviation, CF: care facility, mBP: mean blood pressure, GCS: Glasgow coma scale, qSOFA: quick sequential organ failure assessment score, SOFA: sequential organ failure assessment score, JAAM DIC: Japanese association for acute medicine disseminated intravascular coagulation

Table 2. Result of the Logistic Regression Analysis.

\begin{tabular}{lccccc}
\hline & \multirow{2}{*}{ Partial regression coefficient } & \multirow{2}{*}{ p value } & \multirow{2}{*}{ Odds ratio } & \multicolumn{2}{c}{$95 \%$ confidence interval } \\
& & & & Lower & Upper \\
\hline Need of care & 1.509 & $<0.001$ & 4.521 & 2.024 & 10.096 \\
GCS & -0.122 & $<0.001$ & 0.885 & 0.838 & 0.935 \\
\hline
\end{tabular}

Model chi square test $\mathrm{p}<0.01$, Hosmer-Lemeshow test $\mathrm{p}=0.296$, Predictive accuracy $64.4 \%$.

GCS: Glasgow coma scale

Group B, $\mathrm{p}=0.002]$. The percentage of patients with a previous illness that affected their ADL was $51.4 \%$ (19/37) in Group A and 28.4\% (19/67) in Group B, showing a significant difference $(\mathrm{p}=0.033)$. The mean number of prescribed drugs was $7.3 \pm 3.9$ in Group A and $5.6 \pm 3.8$ in Group B, showing a significant difference $(\mathrm{p}=0.027)$. The mean GCS was $11.3 \pm 3.9$ in Group A and 12.6 \pm 3.4 in Group B (p= 0.040). There were significant difference in the rate of the patient being the decision-maker regarding their own wellbeing and the rate of aspiration pneumonia, although these were data that could not be obtained in the prehospital phase. In summary, for the prehospital data, there were significant differences in the age, percentage of patients residing in a $\mathrm{CF}$, percentage of patients unable to fully care for themselves, rate of dementia, number of prescribed drugs, previous illness that affected the ADL, and GCS between the two groups. However, there were no significant differences in the gender distribution, $\mathrm{mBP}$, heart rate, respiratory rate, blood temperature, $\mathrm{SpO}_{2}$, qSOFA, SOFA, or JAAM
DIC between the two groups. There was also no significant difference in the in-hospital mortality rate between the two groups (16.2\% in Group A vs. $7.5 \%$ in Group B, p=0.193).

The results of the logistic regression analysis are shown in Table 2. In this analysis, the inability to fully care for oneself and a low GCS were related to not wishing to receive intensive treatment. The model for predicting not wishing to receive intensive treatment was as follows:

\section{$\mathrm{P}=1 /[1+\exp (-1 \times$ score $)]$}

Score $=1.509 \times($ with care $)+(-0.122) \times$ GCS

(Model $\chi^{2}$ test $\mathrm{p}<0.001$, Hosmer-Lemeshow test $\mathrm{p}=0.296$, Predictive accuracy $64.4 \%$ ).

\section{Discussion}

In elderly patients with an RTI, the absence of a WIT does not mean that there is no possibility of survival; indeed, to our surprise, we noted no significant difference in the survival rate between the two groups. We initially be- 
lieved that the survival rate of group B would be better than that of group A, similar to the report by Oshitani et al. on do-not-resuscitate (DNR) patients (although group A in our study included not only 'no resuscitation' but also no other intensive treatments) (8). Our results are similar to those of Wall et al. (9). The reason for these findings may be that elderly patients lack a sufficient physical capacity to receive intensive treatment, or Oshitani's results were biased in that the medical staff did not provide medical treatment to patients with a DNR without realizing it, or only type II error.

Having an $\mathrm{AD}$ and advance care plan improve the quality of end-of-life care and increase the satisfaction of elderly patients and their families (10). Several studies have reported that over $50 \%$ of aged nursing home residents have an AD in the United States $(11,12)$ and in Germany $(13)$.

Given that $37.5 \%$ of the patients or their families in the present study refused intensive treatment, we deemed it unnecessary to transfer these patients to an academic medical center, as they had no desire to receive intensive treatment. These results suggest that many Japanese people have a similar attitude to those in Western countries regarding the end of their life. The low rate of ADs among Japanese elderly people may be due to the low organ transplantation rate in Japan (14). Therefore, in Japan, establishing ADs and advance care planning is not likely to become commonplace among elderly people.

We believe that having an $\mathrm{AD}$ is the best way to decide on the therapeutic principle for elderly patients, although elderly people with an AD are quite rare in Japan. Since most Japanese people do not have an AD, Japanese doctors and emergency medical services and staff members at CFs cannot rely on an $\mathrm{AD}$ as a guideline. We therefore conducted the present study to develop a model for predicting the wish not to receive intensive treatment based on the prehospital data. We feel that our findings will be useful in aging societies such as Japan.

Before conducting this analysis, we believed that age and the presence of dementia would be important variables, as a previous report found that the age was an independent predictor of a DNR status (15). As shown in Table 1, there were significant differences in the age and rate of dementia between the two groups. However, through our stepwise logistic regression analysis, we found that an inability to care for oneself fully was a stronger predictor of no WIT. While the percentage of patients who are unable to care for themselves fully is naturally higher among older patients and those with dementia, we feel that the inability to care for oneself fully is suitable as a variable in our model because this factor is more easily comprehensible by staff of emergency medical services than age or dementia. We also believe that our model is adequate because we previously found that elderly people with a low GCS have a higher mortality rate than those with a higher GCS (16). However, the responsible person or caretaker is usually a health aide or the elderly person's child, and the decision-maker is also usually the elderly person's child. In addition, in some cases, the health aides or children are themselves elderly. Therefore, fatigue among caregivers may have prompted the responsible person to decline intensive treatment. In Japan, medical treatment without an AD in elderly people may be for the good of the patients' family and not for the patients themselves. These are characteristics of Japanese medicine among elderly patients and points that need to be improved.

We hope that these findings will prove useful for hospital selection by emergency medical services and staff of CFs. We also hope that physicians practicing at non-tertiary hospitals do not decline to treat patients with a low GCS or who are unable to care for themselves fully because of the severity of their disease. When the WIT of such patients become obvious after admission to a non-tertiary hospital, the patient should promptly be transferred to a tertiary hospital. A multi-center study will hopefully ameliorate such issues.

\section{Limitations}

This was a retrospective study performed at only one institution in a relatively small population. We were unable to analyze patients who were transferred to another hospital by emergency services. Furthermore, the logistic model's predictive accuracy was not very high. The predictive accuracy may be improved if more patients are enrolled in the study; therefore, a multi-center study including community emergency services will be necessary.

\section{Conclusions}

We developed a model to predict the wish to receive intensive treatment among elderly patients with RITs. In a society such as Japan, where many patients lack an AD, elderly patients who are unable to care for themselves and who have a low GCS in the prehospital stage are likely not to want intensive treatment.

The authors state that they have no Conflict of Interest (COI).

\section{References}

1. Fire and Disaster Management Agency in Japan. Annual Report in 2016 [Internet]. [cited 2017 Mar. 2]. Available from: http://www.fd ma.go.jp/neuter/topics/kyukyukyujo_genkyo/h28/01_kyukyu.pdf (in Japanese)

2. Fukuhara T. Restriction of medical treatment for patients with severe persistent consciousness disturbance: irrespective of the desire "to live as long as possible". Seimei Rinri (Bioethics) 25: 38-47, 2015 (in Japanese, Abstract in English).

3. Cabinet Office, Government of Japan. White Paper on Aging Society in 2016 [Internet]. [cited 2017 Mar. 2]. Available from: http:// www8.cao.go.jp/kourei/whitepaper/w-2016/zenbun/28pdf_index.ht $\mathrm{ml}$ (in Japanese).

4. Puisieux F, D’Andrea C, Baconnier P, et al. Swallowing disorders, pneumonia and respiratory tract infectious disease in the elderly. Rev Mal Respire 28: e76-e93, 2011.

5. Vincent JL, de Mendonça A, Cantraine F, et al. Use of the SOFA score to assess the incidence of organ dysfunction/failure in intensive care units: results of a multicenter, prospective study. Crit 
Care Med 26: 1793-1800, 1998.

6. Singer M, Deutschman CS, Seymour CW, et al. The third international consensus definitions for sepsis and septic shock (sepsis-3). JAMA 315: 801-810, 2016.

7. Gando S, Saitoh D, Ogura H, et al. A multicenter, prospective validation study of the Japanese association for acute medicine disseminated intravascular coagulation scoring system in patients with severe sepsis. Crit Care 17: R111, 2013.

8. Oshitani Y, Nagai H, Matsui H. Rationale for physicians to propose do-not-resuscitate orders in elderly community- acquired pneumonia cases. Geratr Gerontol Int 14: 54-61, 2014.

9. Wall J, Hiestand B, Caterino J. Epidemiology of advanced directives in extended care facility patients presenting to the emergency department. West J Emerg Med 16: 966-973, 2015.

10. Detering KM, Hancock AD, Reade MC, Silvester W. The impact of advance care planning on end of life care in elderly patients: randomised controlled trial. BMJ 340: c1345, 2010.

11. Jones AL, Dwyer LL, Bercovitz AR, Strahan GW. The National Nursing Home Survey: 2004 overview. Vital Health Stat 13 1-155, 2009.
12. Daaleman TP, Williams CS, Preisser JS, Sloane PD, Biola H, Zimmerman S. Advanced care planning in nursing homes and assisted living communities. J Am Med Dir Assoc 10: 243-251, 2009.

13. Luck T, Rodriguez FS, Wiese B, et al. Advanced directives and power of attorney for health care in the oldest-old-results of the AgeQualiDe study. BMC Geriatr 17: 85, 2017.

14. McConnell JR 3rd. The ambiguity about death in Japan: an ethical implication fororgan procurement. J Med Ethics 25: 322-324, 1999.

15. Messinger-Rapport BJ, Kamel HK. Predictors of do not resuscitate orders in the nursing home. J Am Med Dir Assoc 6: 18-21, 2005.

16. Ishihara K, Hagiwara $S$, Aoki M, et al. Prognostic factors in emergency patients aged 90 years and older. Acute Med Surg 1: 83-87, 2014 .

The Internal Medicine is an Open Access article distributed under the Creative Commons Attribution-NonCommercial-NoDerivatives 4.0 International License. To view the details of this license, please visit (https://creativecommons.org/licenses/ by-nc-nd/4.0/).

(C) 2018 The Japanese Society of Internal Medicine

Intern Med 57: 1989-1993, 2018 